\title{
Obstructing Small Bowel Bezoars Due to an Agar Diet: Diagnosis Using Double Balloon Enteroscopy
}

\author{
Taro Osada, Tomoyoshi Shibuya, Tomohiro Kodani, Kazuko Beppu, Naoto Sakamoto,
} Akihito Nagahara, Toshifumi Ohkusa, Tatsuo Ogihara and Sumio Watanabe

\begin{abstract}
Primary small bowel bezoars are rare and may cause acute abdomen due to small bowel obstruction (SBO). A 70-year-old Japanese woman presented to the emergency room with abdominal pain, nausea and vomiting. The patient reported that she had eaten a large amount of highly-concentrated, agar dissolved in boiling water two days prior to presentation. Double balloon enteroscopy (DBE) revealed that white-colored, hard bezoars were clogged in the jejunum. At surgery, many bezoars were found impacted in the distal jejunum, and enterotomy was performed. The bezoars were elastic hard, crystallized objects. These bezoars were considered to have formed from highly-concentrated, dissolvable agar.
\end{abstract}

Key words: small bowel obstruction (SBO), double balloon enteroscopy (DBE), agar diet

(Inter Med 47: 617-620, 2008)

(DOI: 10.2169/internalmedicine.47.0520)

\section{Introduction}

Primary bowel bezoars are very rare. Small bowel bezoar is an uncommon cause of acute small bowel obstruction (SBO). Bezoars are usually concretions of foreign material found in the stomach and they most often develop in patients who have undergone gastric surgery. Bezoars usually become impacted in the narrowest portion of the small bowel, which is $50-75 \mathrm{~cm}$ proximal to the ileocecal valve, or at the valve itself $(1,2)$. Preoperative diagnosis of SBO due to bezoars is difficult.

Double balloon enteroscopy (DBE), a new insertion method developed by Yamamoto and colleagues in 2001 (3), allows for complete visualization and therapeutic interventions in the entire small bowel and to-and-fro examination. We present here the first reported case of SBO due to primary small bowel bezoars that had formed from highlyconcentrated, dissolvable agar detected by DBE.

\section{Case Report}

A 70-year-old Japanese woman was admitted through the emergency room after sudden onset of abdominal pain, nau- sea and vomiting. She had a history of giving birth by cesarean operation. On examination, her height was $150 \mathrm{~cm}$ and weight was $67 \mathrm{~kg}$. There was slight tenderness in the left lower quadrant of the abdomen. The patient had been diagnosed with hypertension and type 2 diabetes six years earlier, and she has been taking a $\mathrm{Ca}$ channel blocker and meglitinides. Laboratory evaluation on admission revealed a white blood cell count of $13,600 / \mathrm{mm}^{3}$, C-reactive protein of $2.8 \mathrm{mg} / \mathrm{dl}$, fasting blood sugar (FBS) level of $203 \mathrm{mg} / \mathrm{dl}$ and glycosylated hemoglobin (HbAlc) level of $6.7 \%$. Other chemistry and liver function tests were normal. Plain abdominal radiograph was unremarkable. Computed tomography (CT) showed a dilated small bowel loop proximal to the site of the obstruction and intraluminal masses outlined by fluid (Fig. 1). The small bowel loops distal to the obstruction had a normal appearance. The patient reported that she had eaten a large amount of highly concentrated, agar dissolved in boiling water two days prior to the admission. Antegrade DBE (Fujinon EN-450T5/20; Fujinon Corp., Saitama, Japan) was performed and showed that white-colored, hard bezoars were clogged in the distal jejunum (Fig. 2). However, it was difficult to milk and fragment the bezoars. Radiography of the small intestine under DBE showed that bezoars were clogged in a 10-cm segment of the distal jeju-

Department of Gastroenterology, Juntendo University School of Medicine, Tokyo

Received for publication August 13, 2007; Accepted for publication December 25, 2007

Correspondence to Dr. Taro Osada, otaro@med.juntendo.ac.jp 


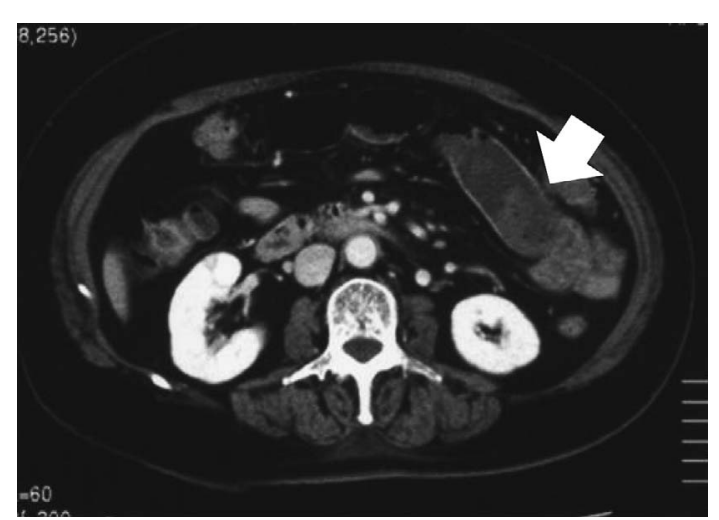

Figure 1. Enhanced computed tomography (CT) scan of the abdomen shows a dilated small bowel loop proximal to the site of the obstruction and intraluminal masses outlined by fluid.

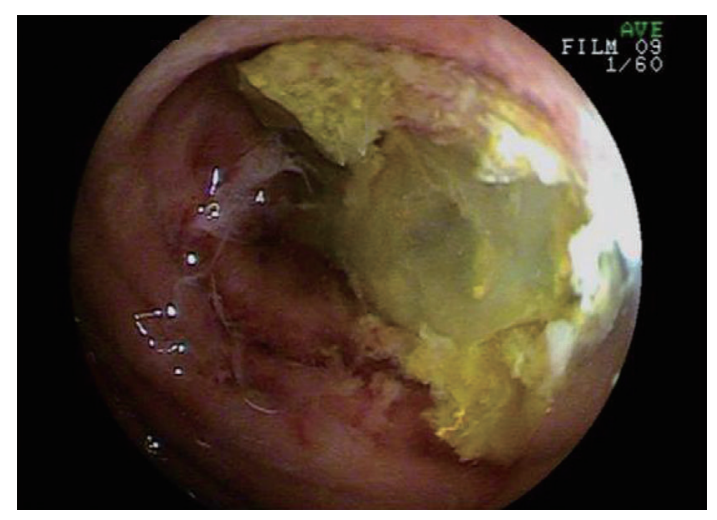

Figure 2. Endoscopic view shows that white-colored bezoars are clogged in the distal jejunum.
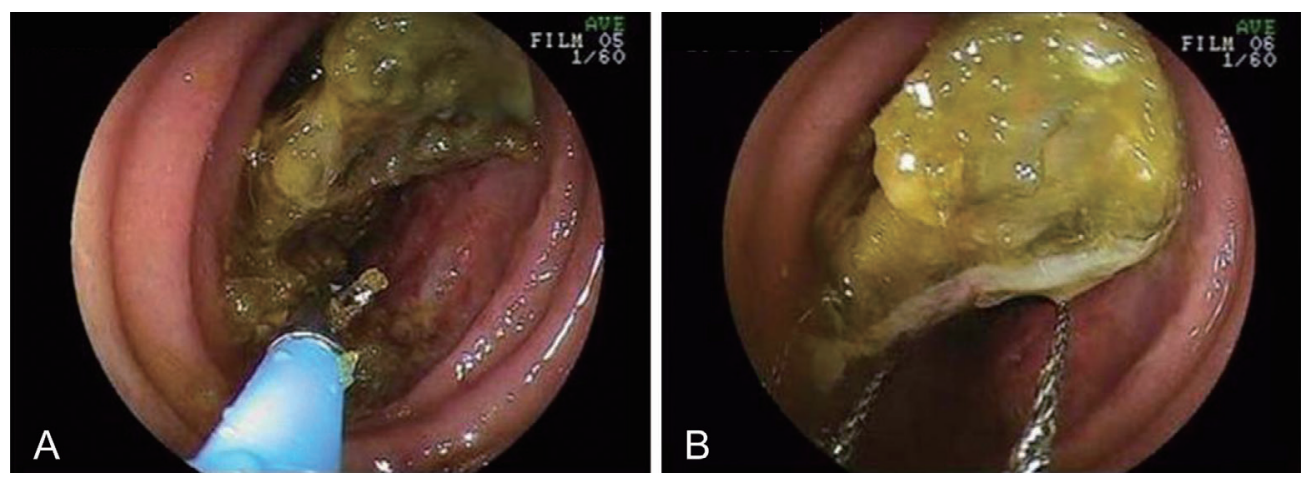

Figure 4. Endoscopic view showing our attempt to smash the bezoars using forceps (A) and a snare $(B)$.

num (Fig. 3). Retrograde DBE was performed two times in an attempt to smash the bezoars to fragments, but the bezoars were too hard and could not be fragmented using a forceps and a snare (Figs. 4A, B). At surgery, many phytobezoars were found impacted in the distal jejunum, and enterotomy with extraction was performed. The resected jejunum had postoperative adhesion of cesarean section. Bezoars of various sizes (max. size $5 \times 4 \times 3 \mathrm{~cm}$ ) were removed. The bezoars were elastic hard, crystallized objects (Fig. 5). Part of a bezoar was analyzed and we found that it was ho-

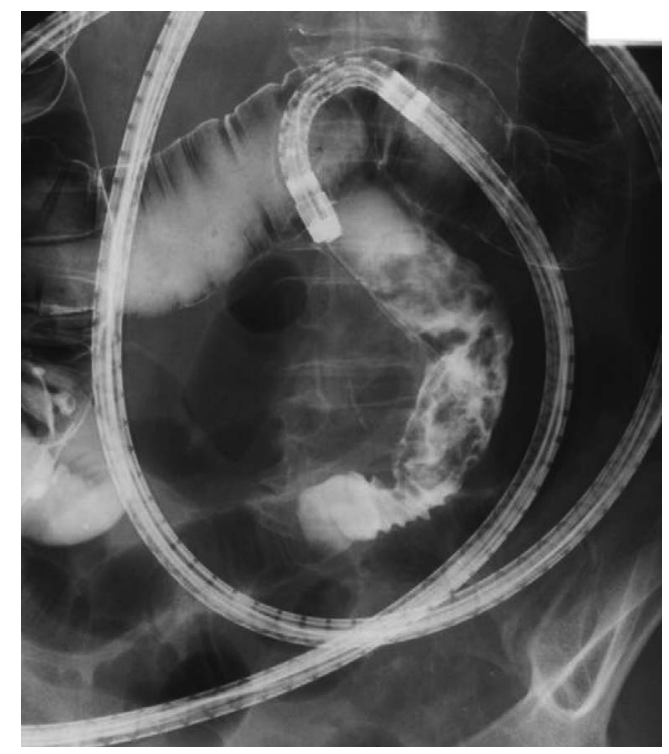

Figure 3. Photo-radiography of the small intestine reveals that about $10 \mathrm{~cm}$ of the distal jejunum was occluded with bezoars. mogeneously composed of a large amount of dietary fibers. These bezoars were therefore considered to have concreted from the highly-concentrated dissolvable agar that the patient had eaten.

\section{Discussion}

Bezoars are usually found in the stomach, but they may also pass into the small bowel. Primary small bowel bezoars are very rare and may cause acute abdomen due to SBO (1, 


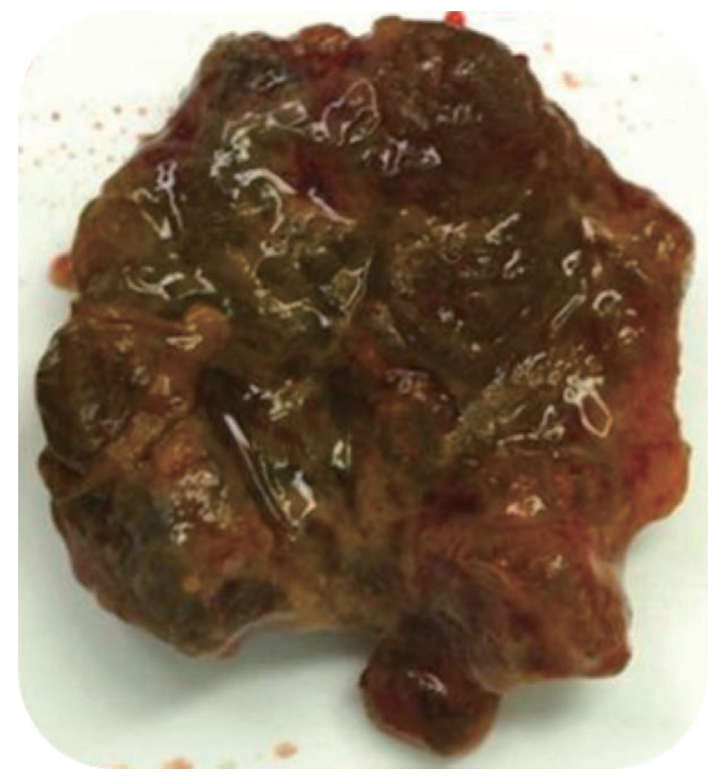

Figure 5. Bezoars of various sizes that had been removed from the jejunum of the patient (max. size, $5 \times 4 \times 3 \mathrm{~cm}$ ). The bezoars were elastic hard.

2). The associated clinical signs and symptoms include vomiting, nausea, abdominal pain, fever, and elevated leukocyte count. Phytobezoars are concretions of fruit and vegetable fibers in the alimentary tract. Other predisposing factors are ingestion of high-fiber foods (4). Agar, a popular food in the traditional Japanese diet called kanten, is produced from red algae and has a high dietary fiber content. It dissolves when heated and forms a gel when cooled. Because it dissolves in water of temperatures above $60^{\circ} \mathrm{C}$, its fiber falls into the water-soluble category. Raw agar is white and semitranslucent. To make jelly, it is boiled in water at a concentration of about $0.7-1 \%$. It was reported that dietary agar caused weight loss and reduction in total cholesterol level in obese patients with impaired glucose tolerance and type 2 diabetes (5). It is thought that dietary fibers inhibit increases in blood glucose by retarding the movement of glucose from the stomach to the small intestine or by obstructing (6) or retarding (7) digestion and absorption of glucose in the small intestine. The present patient had been diagnosed with type 2 diabetes six years earlier, and she sometimes takes dietary agar. SBO occurred due to the formation of bezoars, which had resulted from intake of a large amount of highlyconcentrated, dissolvable agar.

SBO can be diagnosed by various modalities. To date, computed tomography (CT) imaging has been reported to be useful for imaging patients with $\mathrm{SBO}$ as this modality is very effective for determining the presence or absence of SBO as well as the level and cause of SBO (8-12); however, there are only a few reports regarding the CT findings of bezoars associated with $\operatorname{SBO}(13,14)$. DBE is a safe, feasible diagnostic tool that allows high-resolution endoscopic imaging and total enteroscopy, and provides the capability of obtaining tissue for histological studies $(3,15)$. DBE also appears to be a safe and useful diagnostic tool in patients with SBO, and the findings of DBE influence the strategy of therapy in patients in whom the cause of SBO could not be determined by conventional radiography (16). Most bezoars in the stomach are treated by endoscopic destruction or removal. Endoscopic-guided EHL (electrohydraulic lithotripsy) which has gained acceptance in the treatment of biliary and urinary stones, was reported to be a safe and effective for treatment of large and hard gastric bezoars (17). EHL might have been useful for the treatment of small bowel bezoars in this patient. Recently, it was reported that DBE could recover entrapped endoscopy capsules in some patients (1820). In the future, if new devices and methods that can smash or remove primary small bowel bezoars safely are developed, endoscopic therapy using DBE might be more easily performed.

\section{References}

1. Wang PY, Skarsgard ED, Baker RJ. Carpet bezoar obstruction of the small intestine. J Pediatr Surg 31: 1691-1693, 1996.

2. Verstandig AG, Klin B, Bloom RA, Hadas I, Libson E. Small bowel phytobezoars: detection with radiography. Radiology 172: 705-707, 1989.

3. Yamamoto H, Kita H, Sunada K, et al. Clinical outcomes of double-balloon endoscopy for the diagnosis and treatment of small-intestinal diseases. Clin Gastroenterol Hepatol 2: 1010-1016, 2004.

4. Escamilla C, Robles-Campos R, Parrilla-Paricio P, LujanMompean J, Liron-Ruiz R, Torralba-Martinez JA. Intestinal obstruction and bezoars. J Am Coll Surg 179: 285-288, 1994.

5. Maeda H, Yamamoto R, Hirao K, Tochikubo O. Effect of agar (kanten) diet on obese patients with impaired glucose tolerance and type 2 diabetes. Diabetes Obes Metab 7: 40-46, 2005.

6. Gardner DF, Schwartz L, Krista M, Merimee TJ. Dietary pectin and glycemic control in diabetes. Diabetes Care 7: 143-146, 1984.

7. Holt S, Heading RC, Carter DC, Prescott LF, Tothill P. Effect of gel fibre on gastric emptying and absorption of glucose and paracetamol. Lancet 1: 636-639, 1979.
8. Kim JH, Ha HK, Sohn MJ, et al. CT findings of phytobezoar associated with small bowel obstruction. Eur Radiol 13: 299-304, 2003.

9. Frager DH, Baer JW. Role of CT in evaluating patients with small-bowel obstruction. Semin Ultrasound CT MR 16: 127-140, 1995.

10. Megibow AJ, Balthazar EJ, Cho KC, Medwid SW, Birnbaum BA, Noz ME. Bowel obstruction: evaluation with CT. Radiology 180: 313-318, 1991.

11. Fukuya T, Hawes DR, Lu CC, Chang PJ, Barloon TJ. CT diagnosis of small-bowel obstruction: efficacy in 60 patients. AJR Am J Roentgenol 158: 765-769, 1992.

12. Gazelle GS, Goldberg MA, Wittenberg J, Halpern EF, Pinkney L, Mueller PR. Efficacy of CT in distinguishing small-bowel obstruction from other causes of small-bowel dilatation. AJR Am J Roentgenol 162: 43-47, 1994.

13. Ko S, Lee T, Ng S. Small bowel obstruction due to phytobezoar: CT diagnosis. Abdom Imaging 22: 471-473, 1997.

14. Quiroga S, Alvarez-Castells A, Sebastià MC, Pallisa E, Barluenga E. Small bowel obstruction secondary to bezoar: CT diagnosis. 
Abdom Imaging 22: 315-317, 1997.

15. May A, Nachbar L, Wardak A, Yamamoto H, Ell C. Doubleballoon enteroscopy: preliminary experience in patients with obscure gastrointestinal bleeding or chronic abdominal pain. Endoscopy 35: 985-991, 2003.

16. Sun B, Shen R, Cheng S, Zhang C, Zhong J. The role of doubleballoon enteroscopy in diagnosis and management of incomplete small-bowel obstruction. Endoscopy 39: 511-515, 2007.

17. Kuo JY, Mo LR, Tsai CC, Chou CY, Lin RC, Chang KK. Nonoperative treatment of gastric bezoars using electrohydraulic lithotripsy. Endoscopy 18: 386-388, 1999.
18. May A, Nachbar L, Ell C. Extraction of entrapped capsules from the small bowel by means of push-and-pull enteroscopy with the double-balloon technique. Endoscopy 37: 591-593, 2005.

19. Lee BI, Choi H, Choi KY, et al. Retrieval of a retained capsule endoscope by double-balloon enteroscopy. Gastrointest Endosc 62: 463-465, 2005.

20. Miehlke S, Tausche AK, Bruckner S, Aust D, Morgner A, Madisch A. Retrieval of two retained endoscopy capsules with retrograde double-balloon enteroscopy in a patient with a history of complicated small-bowel disease. Endoscopy 2006 Jul 18; [Epub ahead of print].

(C) 2008 The Japanese Society of Internal Medicine http://www.naika.or.jp/imindex.html 